\title{
Linear Spectropolarimetry and the Circumstellar Media of Young and Massive Stars
}

\author{
Jorick S. Vink \\ Armagh Observatory, College Hill, BT61 9DG, Armagh, Northern Ireland, UK
}

\begin{abstract}
Linear spectropolarimetry is a powerful tool to probe circumstellar structures on spatial scales that cannot yet be achieved through direct imaging. In this review I discuss the role that emission-line polarimetry can play in constraining geometrical and physical properties of a wide range of circumstellar environments, varying from the accretion disks around pre-main sequence $\mathrm{T}$ Tauri and Herbig Ae/Be stars, to the issue of stellar wind clumping, and the aspherical outflows from the massive star progenitors of supernovae and long gamma-ray bursts at low metallicity.
\end{abstract}

Keywords: star formation, polarimetry, polarization, T Tauri, Herbig Ae/Be, Luminous Blue Variables, Wolf-Rayet, Be stars, supernovae, gamma-ray bursts

PACS: $95,97,32$

\section{INTRODUCTION}

Massive stars and pre-main sequence (PMS) T Tauri and Herbig Ae/Be stars have comparable astrophysical ages, of a few million years. They also share the ubiquitous presence of circumstellar media (CSM), involving disks and winds responsible for their emission lines, and which can be studied using similar tools. Key issues in both topics of star formation and massive-star evolution involve angular momentum physics, which remains poorly understood.

Future instruments including the JWST and the E-ELT focus on questions relating to the early Cosmos, involving the epoch of reionization, as well as local star formation. However, pivotal properties of both PMS and low-metallicity massive stars remain largely unknown. In the following I argue that linear polarimetry can play a relevant role in removing these uncertainties.

With the detection of long-duration gamma-ray bursts (GRBs) at redshifts up to $\sim 9$ 10, just a few hundred million years after the Big Bang, and the potential detection of super-luminous supernovae ( $\mathrm{SNe}$ ) out to redshifts $>4$ [1] with instruments such as PTF, Pan-STARRS, and LSST, we urgently need to understand the progenitors of these cosmic explosions. Currently we do not.

We know that stars up to $\sim 15 M_{\odot}$ produce hydrogen $(\mathrm{H})$ rich type II SNe [2]. However, the massive progenitors of all other core-collapse SNe types, whether involving H-poor Ibc SNe, interacting IIn SNe [3], or even pair-instability SNe (PISNs), where the entire star is disrupted; all metals are released; and no remnant is left, remain as yet elusive. What is clear is that their evolution towards collapse is driven by mass loss and rotation, which are highly intertwined.

There have been several suggestions that rotation affects both the strength and the latitudinal dependence of their outflows, but in turn stellar winds are thought to remove 
significant amounts of angular momentum, possibly down to masses as low as $\sim 10 M_{\odot}$ [4]. Whether mass loss is latitude dependent, occurring from the pole [5], maintaining rapid rotation [6], or from the equator [7, 8], subject to loss of spin, remains an open question.

Given the requirements on spatial resolution in low $Z$ extragalactic environments, where both GRBs [9] and PISNs [10] have been favoured, because of lower mass-loss rates [11], linear polarimetry may be the only way to resolve these issues.

Questions such as whether certain massive stars in particular redshift bins may make GRBs will ultimately rely on the initial conditions, determined by the process of the star's formation. Whilst many aspects of low-mass star formation seem well established in that there is at least a paradigm describing the physical properties of the optically visible T Tauri stars: "magnetospheric accretion", the situation for the intermediate mass $\left(2-15 M_{\odot}\right)$ Herbig Ae/Be stars becomes more patchy, whilst for the most massive stars it is not even clear as to whether they might form via disk accretion at all.

\section{THE TOOL OF SPECTROPOLARIMETRY}

The basic idea of linear spectropolarimetry is very simple. It is largely based on the premise that free electrons in an extended ionized CSM scatter the continuum radiation from the central star, revealing a certain amount of linear polarization. If the projected electron distribution is perfectly circular, e.g. when the CSM is spherically symmetric or when a CS disk is observed face-on, the linear Stokes vectors $Q$ and $U$ cancel, and no polarization is observed (as long as the object is spatially unresolved). If the geometry is not circular but involves an inclined CS disk this is expected to result in some net continuum polarization.

One of the advantages of spectropolarimetry over continuum polarimetry is that one can perform differential polarimetry between a spectral line and the continuum independent of any interstellar or instrumental polarization. The $\mathrm{H} \alpha$ depolarization "line effect" utilizes the expectation that hydrogen recombination lines arise over a much larger volume than the continuum and becomes depolarized (see the left hand side of Fig. 1). Depolarization immediately indicates the presence (or absence) of aspherical geometries, such as disks, on spatial scales that cannot be imaged with the world's largest telescopes.

The basic idea of the technique was explored in the 1970 s by e.g. Poeckert \& Marlborough [12] who employed narrow-band filters to show that Be stars have CS disks as around $55 \%$ of their objects showed the depolarization line effect. It took another couple of decades before interferometry [13] could confirm these early findings. Interestingly, in a recent study of peculiar O stars, [14] did not find evidence for the presence of disks in Oe stars - the alleged counterparts of classical Be stars - although the first detection of a line effect in an Oe star (HD 45314) was reported.

In general we divide the polarimetric data into bins corresponding to $0.1 \%$ polarization, the typical error bar (although the numbers from photon statistics are at least a factor 10 better). We also present the data in $Q U$ diagrams. For the case of line depolarization this translates into a linear excursion from the cluster of points that represents the continuum $\left(P^{2}=Q^{2}+U^{2}\right)$ with the excursion showing the trend when the polarization 

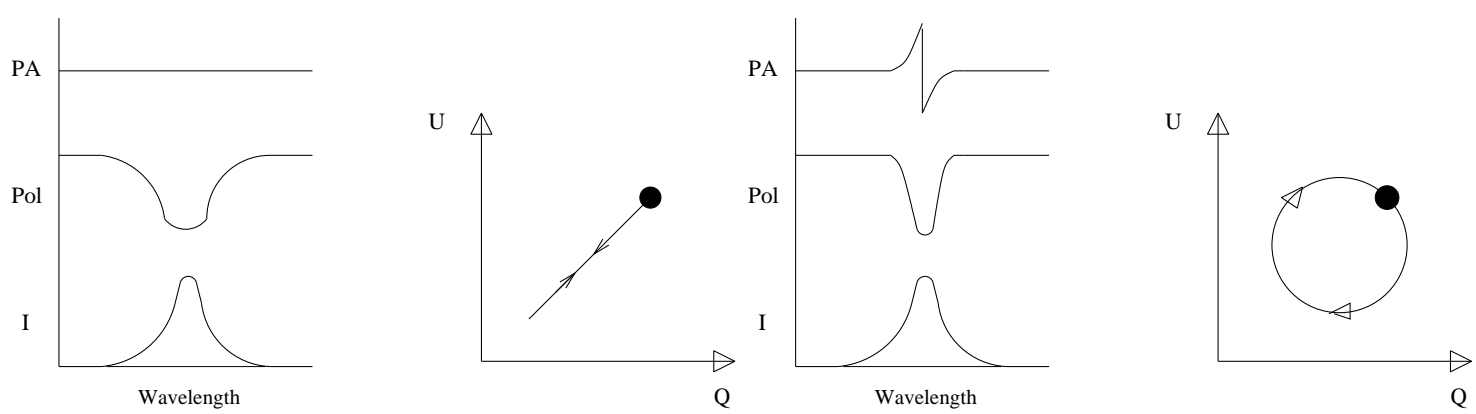

FIGURE 1. Cartoons representing line depolarization (left hand side) and compact line emission scattered off a rotating disk (right hand side) as triplots and $Q U$ diagrams. Stokes $I$ profiles are shown in the lower triplot panels, \% Pol in the middle panels, and the position angles (PAs) are given in the upper triplot panels. Line depolarization is as broad as the Stokes I emission, while the line polarization is narrow by comparison. Depolarization translates into $Q U$ space as a linear excursion (left hand side), whilst a line polarization PA flip is associated with a $Q U$ loop (right hand side).

moves in and out of line center.

A most relevant quantity involves the detection limit, which is inversely dependent on the signal-to-noise ratio (SNR) and the contrast of the emission line to the continuum. The detection limit $\Delta P_{\text {limit }}$ can be represented by:

$$
\Delta P_{\text {limit }}(\%)=\frac{100}{S N R} \times \frac{l / c}{l / c-1}
$$

where $l / c$ refers to the line-to-continuum contrast. This detection limit is most useful for objects with strong emission lines, such as $\mathrm{H} \alpha$ emission in Luminous Blue Variables (LBVs; [15]), where the emission completely overwhelms underlying photospheric absorption. In general, we aim for an SNR in the continuum of 1000, corresponding to changes in the amount of linear polarization of $0.1 \%$. We should be able to infer asymmetry degrees in the form of equator/pole density ratios, $\rho_{\text {eq }} / \rho_{\text {pole }}$ of $\sim 1.25$, or larger [16], with some small additional dependence on the shape and inclination of the disk [17].

Most of the linear line polarimetry work of the last two decades has indeed concerned line depolarization, but in the following we will see that in some cases there is evidence for intrinsic line polarization, predicted by [18] and found observationally in PMS T Tauri and Herbig Ae/Be stars by our group [19, 20, 21, 22, 23]. In such cases line photons are thought to originate from a compact source, e.g. as a result of (magnetospheric) accretion. These compact photons are scattered off a rotating disk, leading to a flip in the position angle (PA), and resulting in a rounded loop (rather than a linear excursion) in the $Q U$ diagram (sketched on the right hand side of Fig. 1).

\section{PRE-MAIN SEQUENCE STARS AND THEIR ACCRETION DISKS}

Over the last decade we have surveyed PMS Herbig Ae/Be stars and T Tauri stars mostly using the $4 \mathrm{~m}$ William Herschel Telescope (WHT). These studies involved roughly equal numbers (of over 10) for each subgroup consisting of $\sim 1 M_{\odot}$ T Tauri stars, $\sim 2-3 M_{\odot}$ 


\begin{tabular}{lllrrrr}
\hline Object & Alt. Name & Type & $\begin{array}{r}\text { Disk PA } \\
\left({ }^{\circ}\right)\end{array}$ & $\begin{array}{r}\text { Pol. PA } \\
\left({ }^{\circ}\right)\end{array}$ & $\begin{array}{c}\Delta \text { PA } \\
\left({ }^{\circ}\right)\end{array}$ \\
\hline \hline HAe/Be & & & $7^{1}$ & $93^{2}$ & 86 & $\perp$ \\
HD 200775 & MWC 361 & B2 & $80^{3}$ & $168^{2}$ & 88 & $\perp$ \\
MWC 147 & V700 Mon & B6 & $77^{5}$ & $164^{6}$ & 87 & $\perp$ \\
HD 45677 & FS CMa & B2 & & 74 & \\
BD +40 4124 & V1685 Cyg & B3 & $110^{7}$ & $36^{2}$ & 74 & $\perp$ \\
MWC 1080 & V628 Cas & B0 & $55^{7}$ & $75^{2}$ & 20 & $\|$ \\
CQ Tau & HD 36910 & F3 & $120^{8}$ & $20^{9}$ & 80 & $\perp$ \\
MWC 480 & HD 31648 & A3 & $150^{7}$ & $55^{9}$ & 85 & $\perp$ \\
AB Aur & HD 31293 & A0 & $79^{10}$ & $160^{9}$ & 81 & $\perp$ \\
HD 179218 & MWC 614 & A0IVe & $23^{11}$ & $\sim 45^{12}$ & 22 & $\|$ \\
T Tauri & & & & & & \\
RY Tau & HD 283571 & F8 & $62^{13}$ & $163^{9}$ & 79 & $\perp$ \\
SU Aur & HD 282624 & G2 & $127^{14}$ & $130^{9}$ & 3 & $\|$ \\
FU Ori & HBC 186 & G3 & $47^{15}$ & $45^{9}$ & 2 & $\|$ \\
GW Ori & HD 244138 & G5 & $56^{16}$ & $(60)^{9}$ & 4 & $\|$ \\
DR Tau & HBC 74 & K5 & $128^{17}$ & $120^{9}$ & 8 & $\|$ \\
\hline
\end{tabular}

FIGURE 2. Comparison of polarization PAs versus imaged disk PAs for a selection of binary PMS from [24]. See [22] for a larger sample of polarization PAs.

Herbig Ae stars and $10-15 M_{\odot}$ early Herbig Be stars. Typical examples can be found in Fig. 3. The PA angles derived have been compared to those obtained from other techniques and generally show great consistency (see e.g. Fig. 2 taken from [24, 22].

\section{Herbig Ae/Be and T Tauri PMS data}

These data may provide insight into the accretion geometry as a function of stellar mass. One might perhaps expect the physics of radiation pressure to dominate the highmass range, whilst the effects of stellar magnetism could dominate the later spectral types. Furthermore, there might be a transition in accretion physics between the T Tauri and the Herbig $\mathrm{Ae} / \mathrm{Be}$ stars, as this is the location in the stellar Hertzsprung-Russell diagram where there is a boundary between the early-type OBA stars with their radiative outer layers, and the convective mantles of the later spectral types.

Intriguingly, the linear spectropolarimetry data reveal a story that is very different: the Herbig Ae stars resemble the T Tauri data and have little in common with the early Herbig Be stars. The Herbig Be stars show line depolarization in 7/12 cases, which is consistent with the presence of small-scale electron scattering disks around all of them. This is a very similar incidence rate as was found for the classical Be stars, and these data are consistent with direct disk accretion for the more massive stars [25, 19].

The question is how to interpret the PA flips seen in the Herbig Ae and T Tauri stars. When we plot the data in $Q U$ space we find $Q U$ loops (e.g. Fig. 4). 

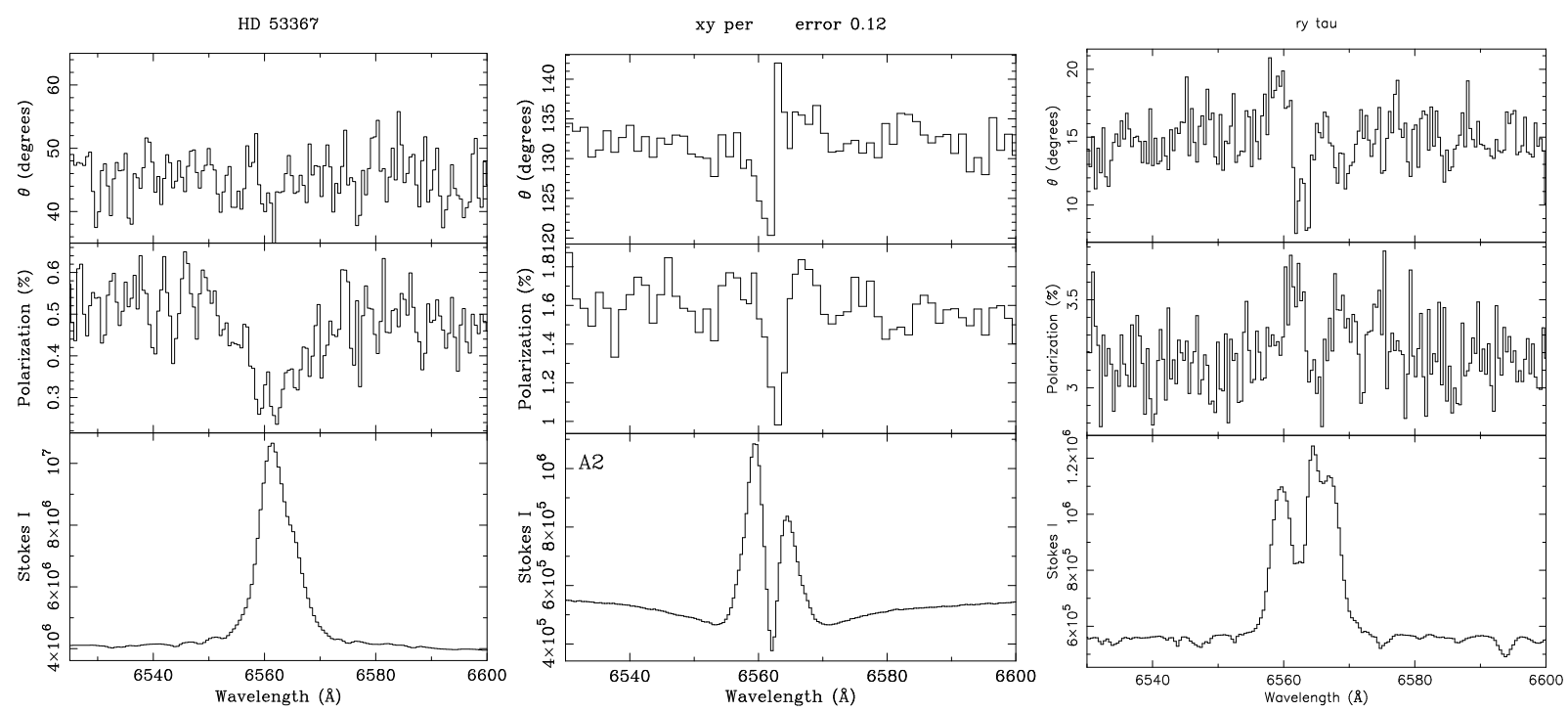

FIGURE 3. H $\alpha$ spectropolarimetry of a Herbig Be object (left), a Herbig Ae star (middle) and a T Tauri star (right). As discussed in the text, the Herbig Ae stars show larger spectropolarimetric resemblance to T Tauri stars than to Herbig Be stars.

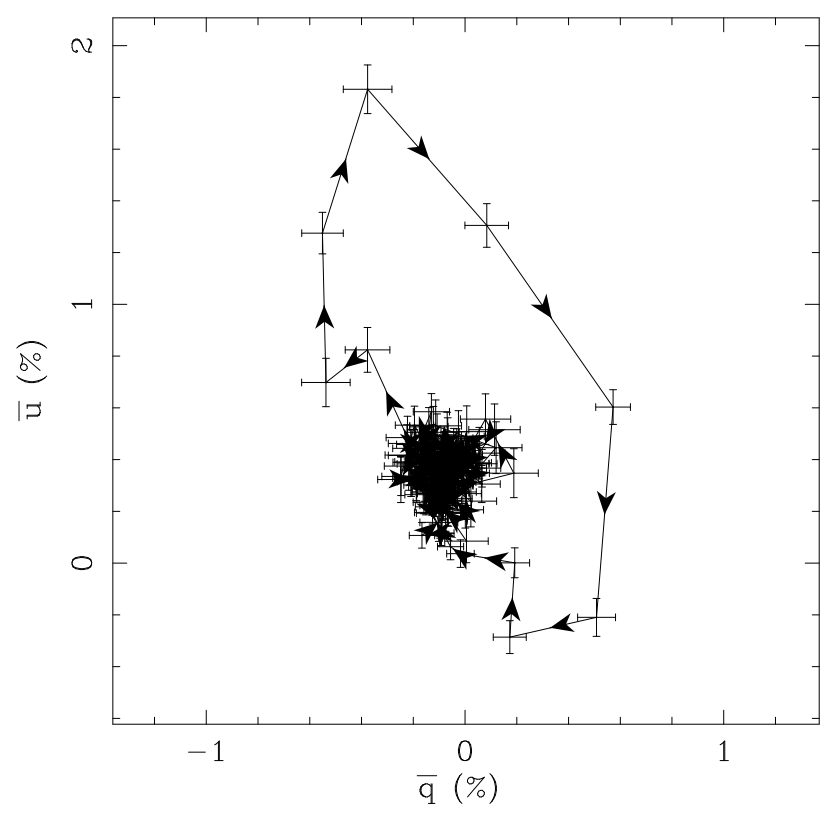

FIGURE 4. A loop in the $Q U$ diagram of the Herbig Ae star MWC 480.

\section{Monte Carlo modelling}

Motivated by the almost ubiquitous incidence of $Q U$ loops in T Tauri and Herbig Ae stars $([19,20,22]$ but see also [26] for alternative findings/interpretations), we decided to develop numerical polarization models of line emission scattered off Keplerian rotating disks [21] using the 3D Monte Carlo code TORUs [16], both with and without a disk in- 

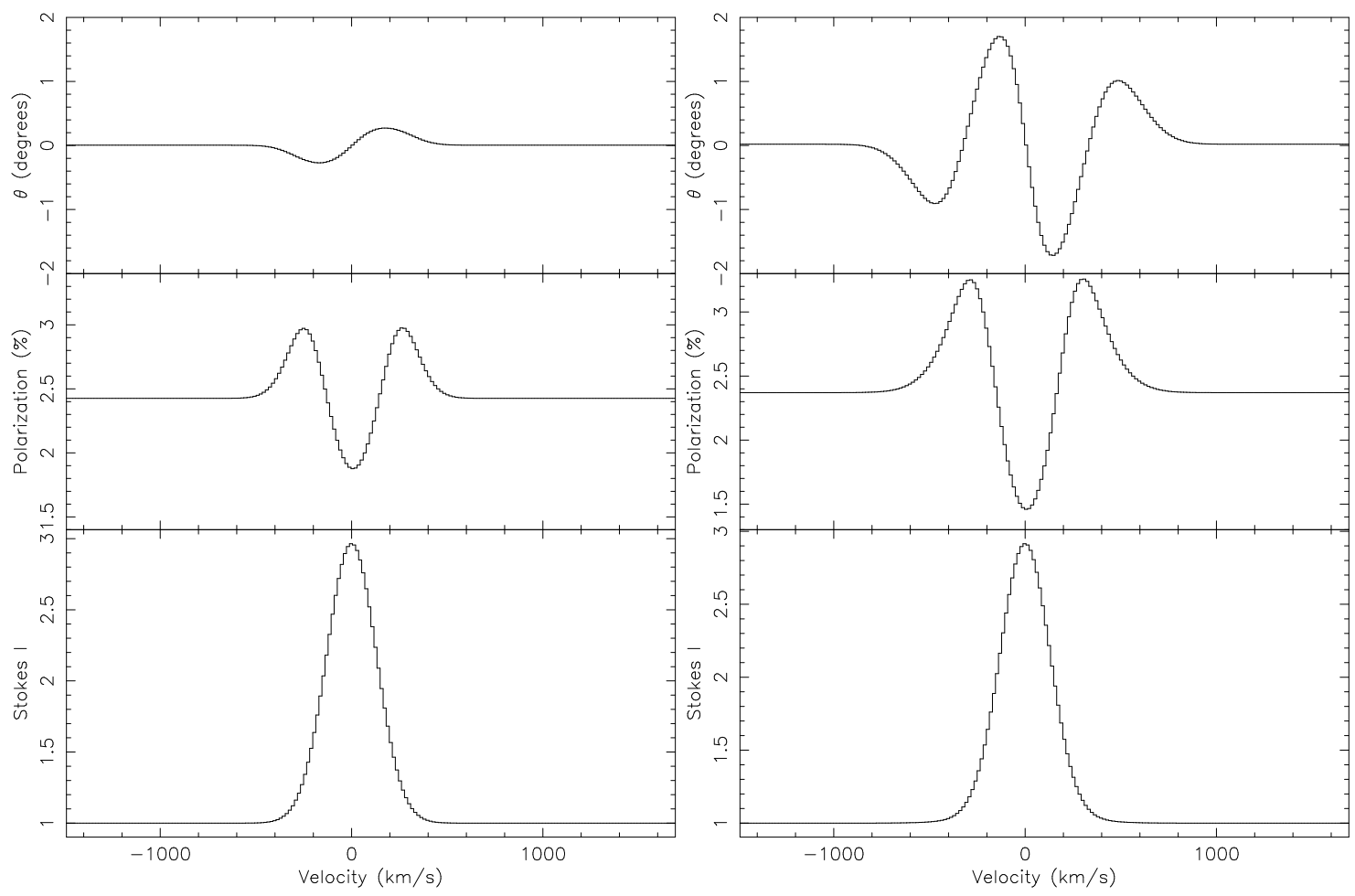

FIGURE 5. Monte Carlo line polarimetry predictions for the case of a disk with an inner hole (left hand side) and without an inner hole - a result of the finite size of the star (right hand side). From [21].

ner hole. Figure 5 shows a marked difference between scattering off a disk that reaches the stellar photosphere (right hand side), and a disk with a significant inner hole (left hand side). The single PA flip on the left-hand side is similar to that predicted analytically [18], but the double PA flip on the right-hand side - associated with the undisrupted disk - came as a surprise at the time. The effect is the result of the geometrically correct treatment of the finite-sized stars that interacts with the disk's rotational velocity field. Our numerical models demonstrate the diagnostic potential of line polarimetry (as opposed to simple depolarization, where no velocity information can be obtained) in determining not only the disk inclination, but also the size of disk inner holes. As far as we are aware linear line polarimetry is as yet the only method capable of determining disk hole sizes on the required spatial scales.

\section{DISKS AND CLUMPS OF MASSIVE STARS}

Line depolarization has been observed in a plethora of massive stars, including $\mathrm{B}[\mathrm{e}]$ supergiants (e.g. [25, 27]), post Red Supergiants [28], and LBVs (e.g. [29]). In all these cases the incidence rate of "line effects" appears to be consistent with the Be star results, i.e. 50-60\%. Furthermore, the measured PA of the line-effect stars shows great consistency with PA constraints from other techniques, providing further proof that the tool is capable of discovering and constraining CS disks. 

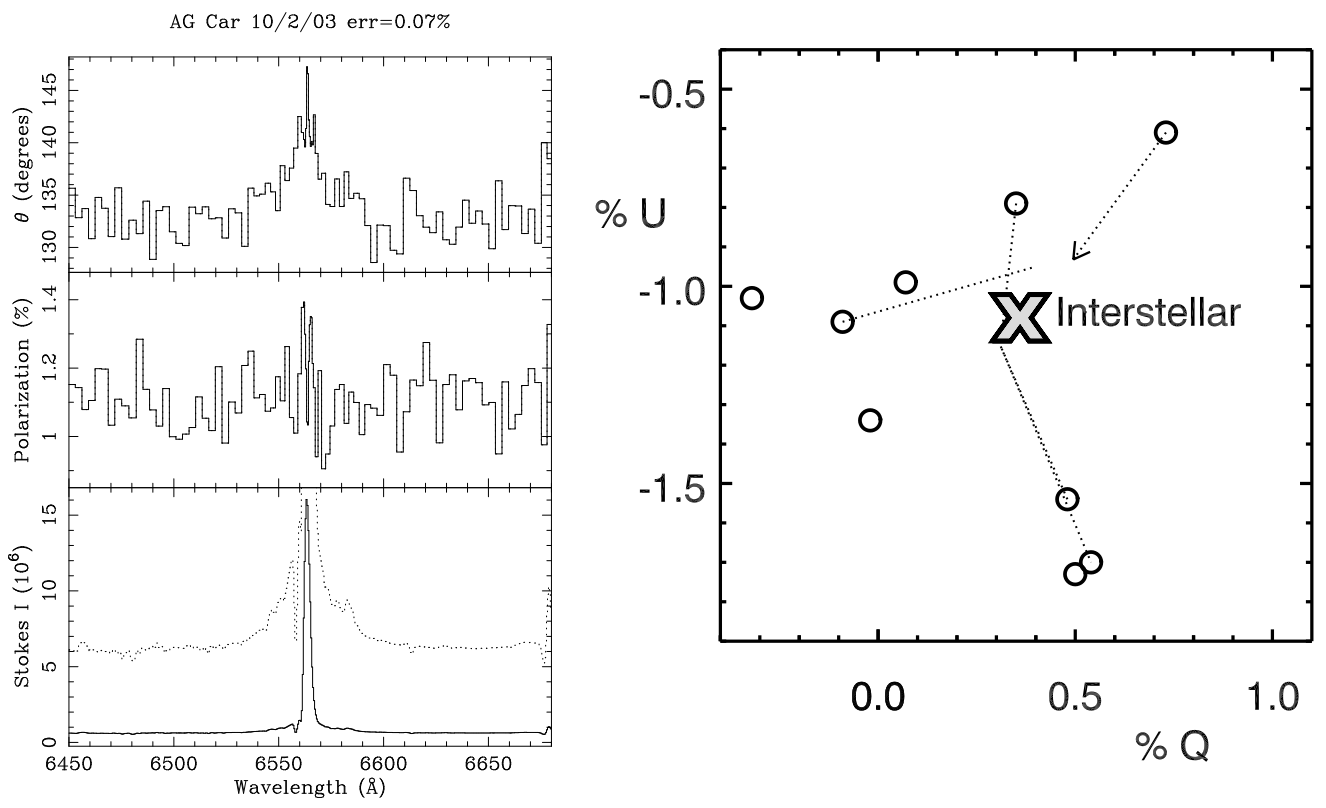

FIGURE 6. H $\alpha$ polarimetry of the Luminous Blue Variable AG Car. The triplot on the left hand side reveals line depolarization. The large cross on the right hand side denotes the measured polarization at line center (constant with time), whilst the open circles represent the continuum measurements that vary with time, indicating wind clumping. See $[15,31]$ for details.

[15] performed a spectropolarimetry survey of LBVs in the Galaxy and Magellanic Clouds and found some surprising results. At first sight the results suggested the presence of disks (or equatorial outflows), as the incidence rate of line effects was inferred to be $>50 \%$. This is notably higher than that of their evolutionary neighbour $\mathrm{O}$ and Wolf-Rayet (WR) stars, with incidence rates of $<25 \%$ [14] and $\sim 15 \%$ [30] respectively. However, when Davies et al. [15] plotted the results of AG Car in a $Q U$ diagram (see Fig. 6) they noticed that the level of polarization varied with time, which they interpreted as the manifestation of wind clumping. Subsequent modelling by [31], [32], and Townsend (Poster this meeting) shows how time-variable linear polarization might become a powerful tool to constrain clump sizes and numbers. These constraints have already been employed in theoretical studies regarding the origin of wind clumping [33] and the effects of wind clumping on predicted mass-loss rates [34].

\section{THE GEOMETRY OF WOLF-RAYET STARS AT LOW METALLICITY AND THE LONG GRB CONNECTION}

Evidence has been accumulating that long GRBs are associated with the deaths of massive stars at low metallicity $Z$ [9]. The next piece of the puzzle is to constrain the progenitors of these explosive events. The currently most popular model is the so-called "collapsar model", where a rapidly rotating compact star collapses to a black hole. This progenitor star is likely a hydrogen-free WR star, but it is currently unclear whether such an object is the result of single star or binary evolution. In both scenarios the crucial 


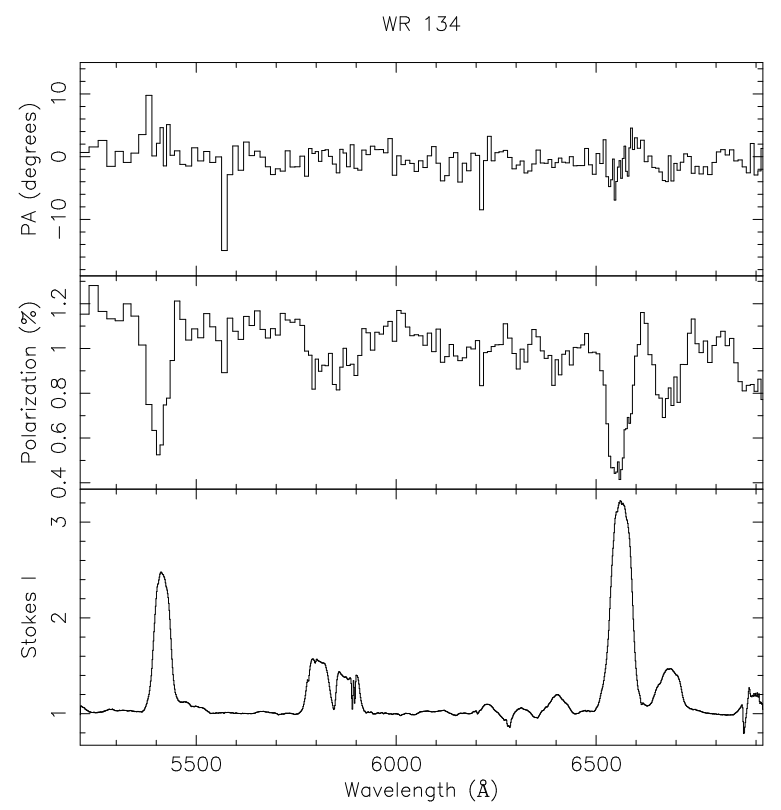

FIGURE 7. WHT data of the Galactic Wolf-Rayet star WR 134. The data are taken from [30].

aspect of low $Z$ is the reduced amount of angular momentum loss due to weaker stellar winds at low metal content. A recent breakthrough in the metallicity dependence of stellar winds from massive stars has been the finding that WR winds are expected to scale with the iron content (Fe) of the host galaxy [11], and not on self-enrichment of metals such as carbon, as was generally assumed previously

Although it has become more firmly established that there is a low $Z$ bias in the occurrence of long GRBs, what is less clear is whether low $Z$ is an absolute requirement. With a bias in $Z$ that is low enough, one might envision a situation in which all massive single stars below a certain threshold $Z$ could make GRBs. Alternatively, the small fraction of GRB supernovae might require special circumstances to ensure that only a small fraction of core-collapse supernovae occur in conjunction with a long GRB.

We decided to test the physical criteria of GRB progenitor models empirically using ESO's FORS spectropolarimeter on the Very Large Telescope (VLT). If the WR mass-loss metallicity dependence and the subsequent inhibition of angular momentum removal are the key to explain the high occurrence of GRBs at low $Z$, WR stars in the Magellanic Clouds (MCs) should on average be spinning faster than those in the Galaxy. Vink [35] therefore performed a linear spectropolarimetry survey of WR stars in the low $Z$ environment of the LMC and found that $\sim 15 \%$ of LMC WR stars might show the sign of rapid rotation, as only 2 out of 13 of them show a significant amount of linear polarization. This incidence rate equals that of the Galactic WR survey by Harries et al. (1998). The LMC data presented in [35] may either suggest that the metal content of the LMC is high enough for the WR winds to remove the angular momentum, and single star progenitors may be constrained to an upper metallicity of that of the LMC at $~ 50 \%$ solar, or alternatively, rapid rotation of WR stars may only be achieved for objects that are the products of a special kind of (e.g. binary) evolution. 
We emphasize that thus far, the best-fitting results (using both Kolmogorov-Smirnov tests and Monte Carlo simulations) of the Harries et al. (see Fig. 7 for an example) sample were obtained if the majority of Galactic objects were spherically symmetric slow rotators, whilst the $15-20 \%$ minority represent the more rapid rotators with large intrinsic polarizations (with values exceeding $0.3 \%$ ). Harries et al. therefore concluded that the inferred axi-symmetries are only present for the most rapidly rotating WR stars.

\section{SUMMARY}

In this review I have attempted to show that linear spectropolarimetry can become a powerful tool to study the geometries and sizes of circumstellar disks and clumps. Moreover:

- Herbig Ae/Be stars were found to possess accretion disks on the smallest spatial scales.

- Contrary to common wisdom, a transition in the HR diagram was found between the Herbig Ae and the Herbig Be stars. The Herbig Be data are consistent with disk accretion at high stellar masses, whilst the Ae data are more reminiscent of T Tauri physics.

- Time-variable polarimetry data suggests that wind clumping rather than the presence of disks is responsible for the high levels of polarization in LBVs.

- Wolf-Rayet stars are found to be equally spherically symmetric in the LMC at half solar metallicity than in the Galaxy at solar metallicity.

\section{ACKNOWLEDGMENTS}

I am extremely grateful to Rico Ignace and the other organizers fur putting together such a great conference in a perfect location, as well as for their generous support. I am also indebted to my senior and junior specpol collaborators: Janet Drew, Tim Harries, Rene Oudmaijer, Ben Davies, Joe Mottram, Mitesh Patel, and Hugh Wheelwright.

\section{REFERENCES}

1. R. M. Quimby, et al., Nature 474, 487-489 (2011).

2. S. J. Smartt, et al., MNRAS 395, 1409-1437 (2009).

3. J. L. Hoffman, et al., ApJ 688, 1186-1209 (2008).

4. J. S. Vink, et al., $A \& A$ 512, L7+ (2010).

5. S. P. Owocki, et al., ApJ 472, L115+ (1996).

6. G. Meynet, and A. Maeder, A\&A 464 L11-L15 (2007).

7. J. E. Bjorkman, and J. P. Cassinelli, ApJ 409 429-449 (1993).

8. F. I. Pelupessy, et al., $A \& A 359$ 695-706 (2000).

9. S. E. Woosley, and A. Heger, ApJ 637 914-921 (2006).

10. N. Langer, et al., A\&A 475 L19-L23 (2007).

11. J. S. Vink, and A. de Koter, A\&A 442 587-596 (2005).

12. R. Poeckert, and J. M. Marlborough, ApJ 206 182-195 (1976).

13. S. M. Dougherty, and A. R. Taylor, Nature 359 808-810 (1992). 
14. J. S. Vink, et al., $A \& A \mathbf{5 0 5} 743-753$ (2009).

15. B. Davies, et al., $A \& A 439$ 1107-1125 (2005).

16. T. J. Harries, MNRAS $315722-734$ (2000).

17. J. C. Brown, and I. S. McLean, A\&A 57 141-+ (1977).

18. K. Wood, et al., A\&A 271 492-+ (1993).

19. J. S. Vink, et al., MNRAS 337 356-368 (2002).

20. J. S. Vink, et al., $A \& A 406703-707$ (2003).

21. J. S. Vink, et al., $A \& A 430$ 213-222 (2005a).

22. J. S. Vink, et al., MNRAS 359 1049-1064 (2005b).

23. J. C. Mottram, MNRAS 377 1363-1374 (2007).

24. H. E. Wheelwright, et al., $A \& A 532$ A28+ (2011).

25. R. D. Oudmaijer, and J. E. Drew, MNRAS 305 166-180 (1999).

26. D. M. Harrington, and J. R. Kuhn, ApJ 667 L89-L92 (2007).

27. A. Pereyra, et al., $A \& A 508$ 1337-1341 (2009).

28. M. Patel, et al., MNRAS 385 967-978 (2008).

29. R. E. Schulte-Ladbeck, et al., ApJ 429 846-856 (1994).

30. T. J. Harries, et al., MNRAS 296 1072-1088 (1998).

31. B. Davies, et al., $A \& A 469$ 1045-1056 (2007).

32. Q. -K. Li, et al., RAA $9558-576$ (2009)

33. M. Cantiello, et al., A\&A 499 279-290 (2009).

34. L. E. Muijres, $A \& A \mathbf{5 2 6}$ A32+ (2011).

35. J. S. Vink, $A \& A 469707-711$ (2007). 
M. Tanaka: Can you study the relation between PAs derived from polarization and those from the image for WRs?

JSV: If the WR nebula has a preferred axis, the answer would be yes. However, many of the observed WR nebula actually appear to be spherical. Furthermore, the total number of line-effect WR stars is very small, and I fear that one would end up in the low-number statistics regime. For pre-main sequence stars, the correlation between our polarization PAs and those derived from images has been very encouraging.

J. Brown: Regarding clump formation sites, remember (Brown's Theorem! Isle aux Coudres conference) that creating (smallish) clumps by redistribution (e.g. condensation) of wind gas above the photosphere does NOT introduce any polarization. This is because the clump polarization is canceled by the cavity polarization.

JSV: I agree, and this suggests that the clumps cannot form in the wind, but they are probably already present in the photosphere. This is consistent with recent clumpformation scenarios that invoke the Fe opacity bump (Cantiello et al. 2009).

J. Cassinelli: Again in regards to the polarization-Z connection for Wolf-Rayet stars, the higher the $Z$ the larger the M-dot. If a high-Z WR star has a disk it would also have a strong polar wind. As shown by Taylor \& Cassinelli (1992), there can be strong depolarization caused by the polar component of the wind. E.g., if the polar ejecta has an M-dot of only 1/10 that in the equator, it will cancel out the polarization completely. So physical geometry cancellation is also an important aspect of the total polarization.

JSV: Yes geometric cancellation can certainly occur, and the absence of polarization should not be taken as evidence for absence of asymmetry, but only as absence of evidence.

K. Nordsieck: On Wolf-Rayets, it has always struck me that the ones that are polarized are very polarized, and there is no in between. I suspect this is because the WR class is so heterogeneous. Maybe all WR stars of a particular sub-class are polarized.

JSV: It is true that the WR class is very heterogeneous, but it does not seem the be the case that objects from one particular sub-class are predominantly polarized, whilst members from other sub-classes are not. Still, until now we have only been able to study small samples, and it would thus make little sense to divide them up any further into sub-groups (such as WN/WC, early/late, single/binary, etc.). Hopefully, it will become possible in the near future to study significantly large sub-samples!

J. Hoffman: Have you seen any of the work on AGN/Seyfert galaxy line polarization? Some of these objects show similar line effects (e.g. Smith et al. 2005). Q-U loops also show up in many supernovae.

JSV: Yes, Smith et al. (2005) found $Q U$ loops in Seyfert galaxies, which may be related to rotating accretion disks. For the $Q U$ loops in $\mathrm{SNe}$ one probably needs to consider alternative explanations.

D. Leonard: So, WR stars with Z $>0.5$ Zsun are found to have spherical outflows. What's the latest on the metallicity of host galaxies of GRBs? 
JSV: Whilst a low metallicity host is not an absolute requirement, there appears to be a strong preference for long GRBs to occur in low metallicity host galaxies, at least as far as I am aware (but my extragalactic knowledge is limited!).

R. Ignace: In principle, the strong forbidden lines of WR stars can help constrain geometry. Spherical winds produce flat-top lines from the large scale wind, but deviations from flat-top suggest non-spherical flow. WR 134 is an example that is polarized and also has somewhat double-horned emission lines.

JSV: I agree, and I think it is extremely exciting and worthwhile to look for correlations between Stokes $Q U$ polarization versus Stokes $I$ intensity indicators! 